\title{
Tagging Functional Polymorphism in 3' Untranslated Region of Methylene Tetrahydrofolate Reductase and Risk of Ischemic Stroke
}

\author{
Jiajia Shi ${ }^{\text {Wei Hed }}$ Yu Wang ${ }^{\text {b }}$ Jiajia Huac \\ aDepartment of Rehabilitation Medicine, Kunshan Rehabilitation Hospital, Suzhou, Jiangsu, 'binternal \\ Medicine-Neurology, The Sixth People's Hospital of Nantong, Nantong, Jiangsu, 'Department of \\ Rehabilitation Medicine, The Sixth People's Hospital of Nantong, Nantong, Jiangsu, dDepartment of \\ Physical Medicine and Rehabilitation, The Affiliated Jiangyin People's Hospital of Southeast University \\ Medical College, Wuxi, China
}

\section{Key Words}

Bioinformatics • MTHFR • Outcome $\cdot$ Homocysteine $\cdot$ MiRNA

\begin{abstract}
Background/Aims: The association between genetic polymorphisms in the exon or untranslated region of the methylenetetrahydrofolate reductase gene (MTHFR) and the risk of human ischemic stroke (IS) has been well-documented. In this study, we focused on a polymorphism previously screened by high-throughput analysis and on its potential function in patients with IS Methods: This hospital-based case-control study was conducted in 400 patients and 400 healthy volunteers. Genotyping was conducted using TaqMan probes. Potential interactions were predicted by multiple bioinformatics analysis. Relative expression levels of MTHFR were detected confirmed by dual-luciferase assay. Results: The MTHFR polymorphism rs 142884651 was significantly associated with a decreased risk of IS compared with the wild-type GA genotype $(P=0.02)$ and AA genotype $(P=0.001)$. According to bioinformatics analysis and luciferase assay, this polymorphism could attenuate the binding of let-7f and miR-196a ( $P=0.021$ and 0.019 , respectively) leading to the accumulation of MTHFR and degradation of serum homocysteine, and resulting in a better IS outcome. Conclusion: This study demonstrated that the MTHFR rs142884651 polymorphism is associated with a decreased risk of IS and may act as a biomarker of short-term outcome in patients with IS.
\end{abstract}

(C) 2018 The Author(s)

Published by S. Karger AG, Basel

\section{Introduction}

Stroke is the major cause of mortality among the elderly population and a common cause of long-term disability worldwide. Furthermore, the stroke burden continues to increase, J. Shi and W. He contributed equally to this work. 
especially in developing countries such as China $[1,2]$. Ischemic stroke (IS) is a major type of stroke defined as a cerebrovascular accident caused by occlusion of the cerebral arteries leading to sudden neurologic deficit, such as loss of motor function, altered sensations, and cognition or language impairments [3]. Increasing evidence has revealed that both environmental [4-6] and genetic factors might contribute to the occurrence of IS [7].

Methylene tetrahydrofolate reductase (MTHFR) is a key enzyme in the metabolism of circulating homocysteine (tHcy) [8], which is in turn a crucial intermediate in methionine metabolism and causes excessive production of reactive oxygen species (ROS) $[9,10]$. tHcy accumulation can elevate ROS levels by activating MAPK signaling, resulting in cell apoptosis [11]. tHcy has been identified as a risk factor for IS, and is thought to increase thrombotic risk by inducing endothelial injury in the venous and arterial vasculature $[12,13]$. Functional polymorphisms in the exon region of the MTHFR have been shown to be associated with the pathogenesis of IS. For example, the rs1801133 polymorphism was associated with reductions in MTHFR activity of approximately 70\% and 35\% in TT and CT genotype carriers, respectively, leading to increased levels of tHcy, which predicted a poor prognosis among IS patients [14-16]. The A1298C variant resulted in substitution of glutamate (Glu) by alanine (Ala) at codon 429 in the S-adenosylmethionine regulatory domain of the MTHFR protein [17-19].

Traditional analyses of genetic polymorphisms in IS patients have focused on genotype frequencies in patients and control samples. However, micro RNAs (miRNAs) have been shown to function in single nucleotide polymorphisms (SNP)-associated post-transcriptional regulation, and the binding of certain miRNAs could be influenced by different genotypes, resulting in different mRNA expression levels. These functional miRNAs have been reported to play crucial roles in the development and prognosis of human diseases. In this study, we aimed to validate the MTHFR rs142884651 SNP previously screened in a genome-wide association study (GWAS) by our group (data not published). The potential interaction between this polymorphism and miRNA was predicted by multiple bioinformatics analysis.

\section{Materials and Methods}

\section{Clinical information}

400 patients diagnosed with ischemic stroke between July 2010 and October 2016 were enrolled. Diagnosis of patients with ischemic stroke was established clinically and confirmed by using X-ray computed tomography and/or magnetic resonance imaging scans of the brain. The subtype of stroke patients in this study was all the patients with large vessel disease. Another 400 sample collected from healthy control were regarded as control group from this hospital during the same period of time and they were matched to the cases by age and gender. All control individuals visited our hospital for annual health check-ups and were free of ischemic stroke. Diabetes mellitus was defined as a fasting glucose level $\geq 7.0 \mathrm{mmol} / \mathrm{L}$ and/ or $\geq 11.1 \mathrm{mmol} / \mathrm{L} 2 \mathrm{~h}$ after oral glucose challenge, or receiving anti-diabetic medication. Subjects who had smoked more than 10 cigarettes daily for 5 years were considered as smokers. Subjects who drank more than $50 \mathrm{ml}$ of alcoholic beverages daily for 5 years were considered as alcohol drinkers. The National Institutes of Health Stroke Scale (NIHSS) score was used to quantify stroke severity in patients at the time of presentation and discharge. This study was approved by the Ethics Committee of Kunshan Rehabilitation Hospital and The Sixth People's Hospital of Nantong and the study was conducted in accordance with the Helsinki Declaration.

\section{Genotyping}

Blood samples were collected after a $12 \mathrm{~h}$ overnight fasting period and then separated into serum, red blood cells, and buffy coat. Genomic DNA from peripheral whole blood of every validation subject was extracted by using QIAamp DNA blood mini kits (Qiagen, Hilden, Germany) according to the manufacturer's instructions. After quantification, these DNA samples were subjected to DNA genotyping using the TaqMan SNP Genotyping Assay. Briefly, genomic DNA samples of $10 \mathrm{ng}$ each were amplified using PCR in a total volume of $5 \mu \mathrm{L}$ containing TaqMan Universal Master Mix, 80x SNP Genotyping AssayMix, DNase-free water 
and 10-ng DNA. The PCR conditions were $50{ }^{\circ} \mathrm{C}$ for $2 \mathrm{~min}$ and then $95^{\circ} \mathrm{C}$ for $10 \mathrm{~min}$, followed by 40 cycles of $95^{\circ} \mathrm{C}$ for $15 \mathrm{~s}$ and $60^{\circ} \mathrm{C}$ for $1 \mathrm{~min}$ in a 384-well ABI 7900HT Real Time PCR System as described previously [20].

Bioinformatic analysis of miRNAs binding to MTHFR SNP

Bioinformatics software (http://www.bioguo.org/miRNASNP/ and http://bioinfo.life.hust.edu.cn/ miRNASNP2/index.php) was used to detect the candidate SNPs which could affect MTHFR gene regulation via miRNAs.

Cell line, culture, transfection and luciferase reporter assay

Embryonic Kidney 293T cells were obtained from the Chinese Academy of Sciences Cell Bank (Shanghai, China) and cultured in RPMI-1640 (Gibco, Gaithersburg, MD, USA) supplemented with $10 \%$ fetal bovine serum (Invitrogen, Carlsbad, CA, USA) in a humidified incubator with $5 \% \mathrm{CO}_{2}$ at $37{ }^{\circ} \mathrm{C}$. The mimics and control were obtained from Genepharma (Shanghai, China) and transfected into 293T cells by using Lipofectamine 2000 (Invitrogen) according to the manufacturer's protocol.

MTHFR 3'-UTR fragments containing either G or A alleles were amplified using PCR from genomic DNA and cloned into pGL3-promoterless luciferase-based plasmids (Promega, Madison, WI, USA) multiple cloning sites. MTHFR 3'-UTR fragments potentially binding to miRNAs, predicted by bioinformatics analysis or a mutated sequence with the predicted target sites, were cloned using PCR from genomic DNA and inserted into the pGL3 promoter vector (Genscript, Nanjing, China). The cloned plasmids were amplified and verified by DNA sequencing.

For the dual-luciferase reporter assay, 293T cells were plated onto 24-well plates and transfected with 100 ng of pGL3- MTHFR wild, pGL3- MTHFR mutant, and miRNA mimics, respectively, using Lipofectamine 2000 (Invitrogen). A Renilla luciferase vector pRL-SV40 (5 ng) was also co-transfected as a normalization and transfection efficiency control. Each experiment was performed in triplicate and luciferase activity was assessed $48 \mathrm{~h}$ after transfection using the dual-luciferase reporter assay system (Promega). Firefly luciferase activity was normalized to Renilla luciferase activity according to a previous study [21].

\section{Quantitative reverse transcription-polymerase chain reaction ( $q R T-P C R$ )}

The qRT-PCR was performed to determine levels of MTHFR mRNA. In brief, total RNA was isolated from 293T cells using a Trizol reagent (Invitrogen, Carlsbad, CA, USA) and reverse transcribed into cDNA using the TaqMan ${ }^{\circledR}$ Reverse Transcription Kit, according to the manufacturers' instructions (ABI, CA, USA). The cDNA samples were then subjected to qPCR amplification under conditions of $95^{\circ} \mathrm{C}$ for $10 \mathrm{~min}$ followed by 40 cycles of $95{ }^{\circ} \mathrm{C}$ for $30 \mathrm{~s}, 55^{\circ} \mathrm{C}$ for $40 \mathrm{~s}$, and $72{ }^{\circ} \mathrm{C}$ for $30 \mathrm{~s}$, and finally $4{ }^{\circ} \mathrm{C}$ for 30 min using the Realmaster Mix (SYBR Green I) (TAKARA, Dalian, China).

\section{ELISA of plasma homocysteine levels}

Plasma levels of tHcy in patients was assessed using a tHcy ELISA kit (Green Stone, Bern, Switzerland), according to the manufacturers' protocol. The level of plasma tHcy was normalized to the kit standard and the data were summarized as Mean \pm SD and then associated with outcome in ischemic stroke patients.

\section{Statistical analysis}

The association between different genetic variants and ischemic stroke risk was evaluated by calculation of the odds ratios (ORs) and 95\% confidence intervals (CIs) using univariate and multivariate logistic regression analysis. The difference in association of MTHFR mRNA levels with three MTHFR genotypes and of the relative luciferase activity between the wild and mutant genotype were evaluated by using an independent-sample t test. The chi-square test was applied to analyze the difference between clinical characteristics of ischemic stroke patients and healthy controls. Data normality of distribution was calculated by confirming coefficient of skewness and coefficient of kurtosis with 0 and Kolmogorov-Smirnov test with the $\mathrm{P}>0.05$. A goodness-of-fit $\chi 2$-test was performed to estimate Hardy-Weinberg equilibrium of the genotype distribution among control subjects for evaluating possible selection bias and genotyping errors. For the clinical outcome correlation of rs142884651 genotype with IS patients, multigroup comparisons were carried out by one-way analysis of variance (ANOVA) test with post hoc contrasts by Student-Newman-Keuls test. All statistical analyses were two-sided and $\mathrm{P}<0.05$ was considered statistically significant using SPSS 
13.0 (SPSS, Chicago, IL, USA) or SAS software (version 9.1.3; SAS Institute, Cary, NC, USA).

\section{Results}

Clinical characteristics

There was no significant difference in the distribution of patient age or gender between the IS and healthy controls (Table 1). The common risk factors for IS, including smoking, drinking, diabetes, and hypertension were more common in the IS compared with the control group ( $P$ $<0.001$ ). In addition, the traditional biomarkers for cardiovascular and cerebrovascular diseases, including total cholesterol, HDL-C, and LDL-C, were also associated with the occurrence of IS.

MTHFR rs142884651 polymorphism protects against IS

The rs142884651 polymorphism located in the $3^{\prime}$ UTR of the MTHFR gene was screened by GWAS (data not published). We aimed to validate the distribution of MTHFR genotypes among IS patients in this case-control group. The distribution of the rs142884651 polymorphism differed between the 400 IS patients and the control group (Table 2). Logistic regression analysis revealed that MTHFR rs142884651 GA and AA genotypes were significantly associated with decreased risks of developing IS compared with the GG genotype (OR: 0.68; 95\% CI: 0.22-0.87 for GA genotype, and OR: 0.45 ; $95 \%$ CI: $0.29-0.58$ for AA genotype; Table 2 ). A higher number of A alleles was also associated with a decreased risk of IS (OR: 0.57 ; 95\% CI: 0.29-0.69).
Table 1. Clinical characteristics of ischemic stroke patients and healthy controls. a: Student t test for continuous variable, Chi-squared test for categorical variable

\begin{tabular}{|c|c|c|c|}
\hline Characteristics & $\operatorname{Cases}(n=400)$ & Control $(n=400)$ & p value ${ }^{a}$ \\
\hline Age & & & $>0.05$ \\
\hline$>60$ & 349 & 351 & \\
\hline$<60$ & 51 & 49 & \\
\hline Gender & & & $>0.05$ \\
\hline Male & 276 & 244 & \\
\hline Female & 124 & 156 & \\
\hline Smoking exposure & & & $<0.001$ \\
\hline Yes & 301 & 181 & \\
\hline No & 99 & 219 & \\
\hline Drinking exposure & & & $<0.001$ \\
\hline Yes & 279 & 199 & \\
\hline No & 121 & 201 & \\
\hline Diabetes & & & 0.007 \\
\hline Yes & 241 & 203 & \\
\hline No & 159 & 197 & \\
\hline Hypertension & & & $<0.001$ \\
\hline Yes & 311 & 129 & \\
\hline No & 89 & 271 & \\
\hline Total cholesterol(mmol/L) & $4.89(4.21-5.22)$ & $4.78(4.19-5.14)$ & $>0.05$ \\
\hline HDL-C(mmol/L) & $0.86(0.45-1.69)$ & $1.44(1.12-1.68)$ & $<0.001$ \\
\hline LDL-C(mmol/L) & $5.02(4.34-6.22)$ & $2.98(1.98-4.28)$ & $<0.001$ \\
\hline
\end{tabular}

Table 2. Genotype frequencies of the MTHFR rs142884651 in IS patients and healthy controls. ${ }^{\text {aThe }}$ ORs, 95\% CIs and $\mathrm{P}$ value were calculated after adjusting for age, gender, smoking, drinking and other characteristics listed in Table1

\begin{tabular}{lcccccc}
\hline Genotype & \multicolumn{2}{l}{ Cases $(\mathrm{n}=400)$} & \multicolumn{2}{l}{ Controls $(\mathrm{n}=400)$} & OR & P Value \\
& $\mathrm{N}$ & $\%$ & $\mathrm{~N}$ & $\%$ & $(95 \% \mathrm{CI})^{\mathrm{a}}$ & \\
\hline rs142884651 & & & & & & \\
GG & 301 & 75.3 & 278 & 69.5 & 1.00 & \\
GA & 61 & 15.3 & 107 & 26.8 & $0.68(0.22-0.87)$ & 0.02 \\
AA & 38 & 9.4 & 15 & 3.7 & $0.45(0.29-0.58)$ & 0.001 \\
A carrier & 99 & 24.7 & 122 & 30.5 & $0.57(0.29-0.69)$ & 0.002 \\
\hline
\end{tabular}


Fig. 1. Functional miRNAs associated with MTHFR rs142884651. A: Relative expression of MTHFR mRNA in IS patients with different genotypes. B: Luciferase assay. 293T cells were co-transfected with miRNA mimics or control Renilla luciferase vector pRL-SV40 for 48 h. Both firefly and Renilla luciferase activities were measured in the same samples using a luciferase assay kit. Firefly luciferase signals were normalized with Renilla luciferase signals. C: Potential binding site for let-7f and miR-196a on MTHFR. Upper indicates let-7 and lower indicates miR-196a. Data presented as mean $\pm \mathrm{SE} .{ }^{*} \mathrm{P}<0.05$.

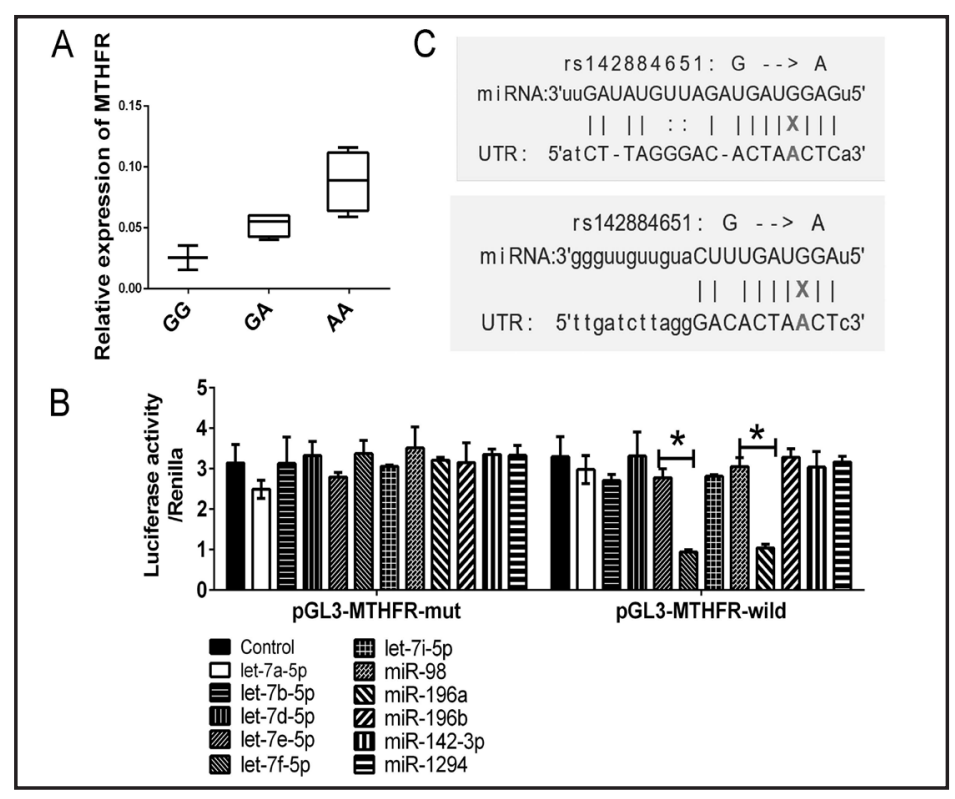

miRNA binding is attenuated by functional SNP

Functional SNPs have been confirmed to affect binding of miRNA to target genes. We detected the expression of different MTHFR genotypes in plasma by RT-PCR. MTHFR expression levels were decreased in patients harboring the A allele, indicating that this SNP located in the $3^{\prime}$ UTR of MTHFR effect its expression $(P=0.023$ ) (Fig. 1A). We therefore performed bioinformatics analysis to determine if MTHFR rs142884651 could interact with miRNAs. Eleven candidate miRNAs were identified by expression correlation values using prediction software (Table 3 ). We cloned these 11 candidate miRNA mimics and transfected them into either MTHFR wild-type or mutant cells. We confirmed binding by luciferase assay and found that the luciferase activity of the G-allele-specific pGL3 construct was significantly suppressed by let-7f and miR-196a ( $P=0.021$ and 0.019 , respectively) (Fig. 1B and C).

\section{MTHFR rs142884651 polymorphism predicts a better outcome in IS patients}

Given that increased MTHFR could promote the metabolism of tHcy, potentially leading to a better prognosis in IS patients, we assessed the association between MTHFR rs142884651 and short-term IS outcome. Initial stroke severity in the acute phase, measured by the NIHSS, differed significantly among the different genotypes. Recovery from IS was measured as the change in NIHSS score from the acute phase to 3 months post-stroke, with a negative value indicating a clinical improvement in IS outcome. The rs142884651 polymorphism predicted a better outcome in IS patients. We also detected levels of MTHFR mRNA expression and circulating tHcy and found that patients with a GA or AA genotype had higher MTHFR expression and decreased levels of tHcy (Table 4).

\section{Discussion}

MTHFR is an important enzyme involved in ROS signaling, especially in relation to tHcy degradation [22]. Indeed, ROS generation is enhanced under ischemic conditions, potentially causing damage to various types of cells, including brain endothelial cells, leading to formation of thromboses and loss of brain function [23]. The current study provided direct evidence to show that the novel MTHFR rs142884651 polymorphism decreased the risk of IS compared with healthy controls. Mechanistically, MTHFR rs142884651 altered the binding of let-7f and miR-196a to negatively regulate MTHFR expression, thus providing a post-transcriptional mechanism for gene regulation and expression.

\section{KARGER}


Increased ROS generation can lead to damage of various types of cells, including brain endothelial cells, causing thrombosis and loss of brain function [24]. MTHFR is an important enzyme in ROS signaling, especially in terms of the degradation of tHcy [22]. Genetic studies have so far revealed more than 40 point mutations in the MTHFR gene, of which C677T (rs1801133) and A1298C (rs1801131) appear to have the most clinical significance. The MTHFR rs1801133 polymorphism involves a $\mathrm{C}$ to $\mathrm{T}$ substitution at position 677 (C677T) resulting in the conversion of alanine to valine [24]. This missense mutation results in reductions in MTHFR activity of approximately $70 \%$ and $35 \%$ in TT and CT genotype carriers, respectively, compared with wild-type MTHFR. MTHFR activity has an adverse effect on tHcy levels. The 677TT allele was also associated with elevated tHcy levels, predominantly in individuals with low plasma folate levels. Higher tHcy concentrations have in turn been shown to have a negative effect on stroke $[16,19]$.

Accumulating evidence suggests that SNPs localized at miRNA-binding sites may affect the binding of miRNAs to their target genes, leading in turn to reduced or increased translation of the target mRNA and altered susceptibility to cancer. For example, the rs2910164 polymorphism harboring the sequence for miR-146a was shown to influence susceptibility to gastric cancer in a Chinese population, while rs4143815 and rs4819388 SNPs in the 3'-UTRs of B7-H1 and B7-H2 genes, respectively, were also associated with the development of gastric cancer [25]. The function of SNPs localized at miRNA-binding sites, also defined as $3^{\prime}$ UTRs, could affect the binding of miRNAs to their targets and thereby suppress or activate the expression of the target genes and alter the susceptibility to cancer [20,26]. In addition, our results showed that MTHFR rs142884651 SNP could affect let-7f and miR-196a binding, leading to low cellular MTHFR

Table 3. Candidate miRNAs associated with MTHFR rs142884651 predicted by bioinformatics analysis.

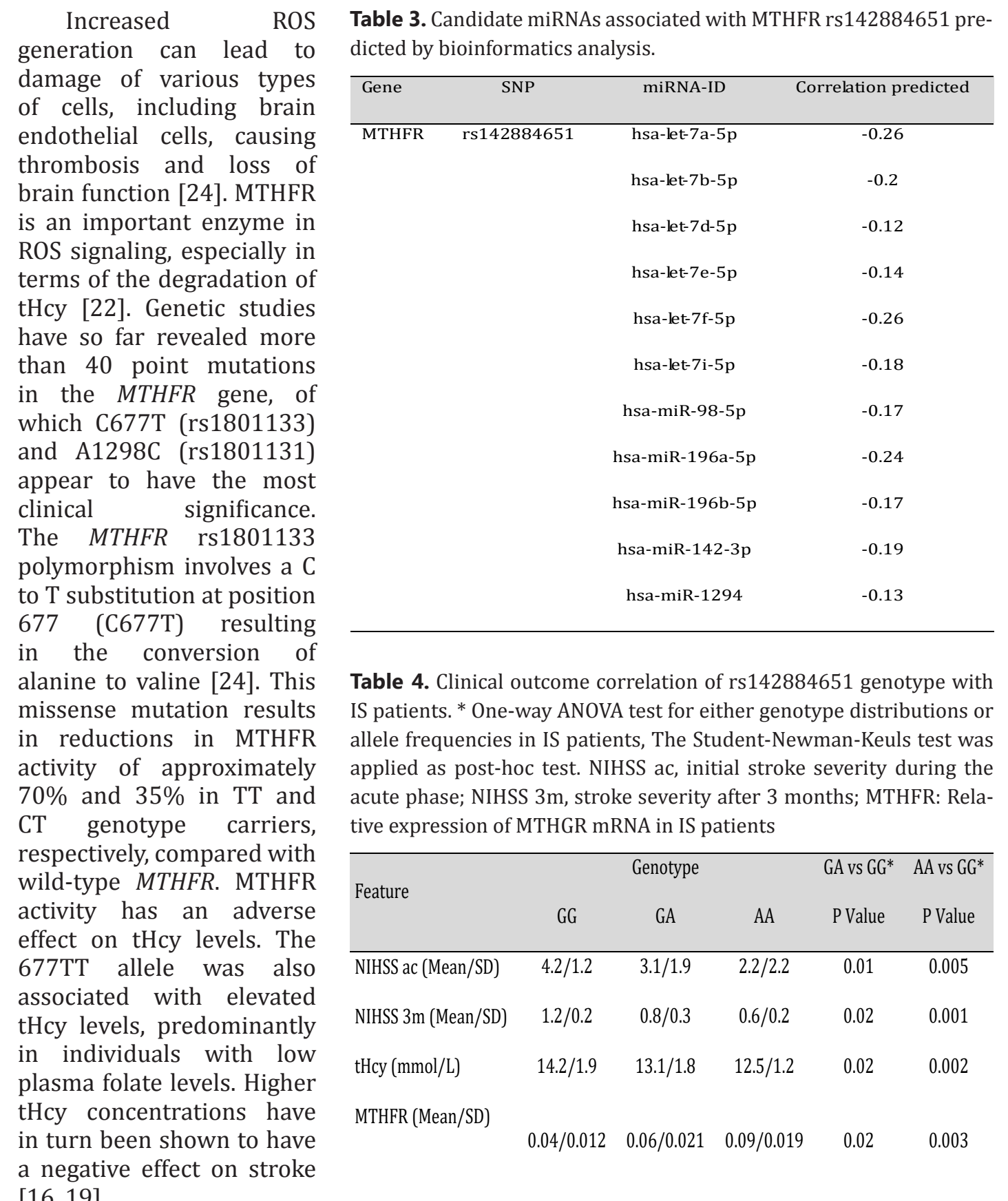

Table 4. Clinical outcome correlation of rs142884651 genotype with IS patients. * One-way ANOVA test for either genotype distributions or allele frequencies in IS patients, The Student-Newman-Keuls test was applied as post-hoc test. NIHSS ac, initial stroke severity during the acute phase; NIHSS 3m, stroke severity after 3 months; MTHFR: Relative expression of MTHGR mRNA in IS patients

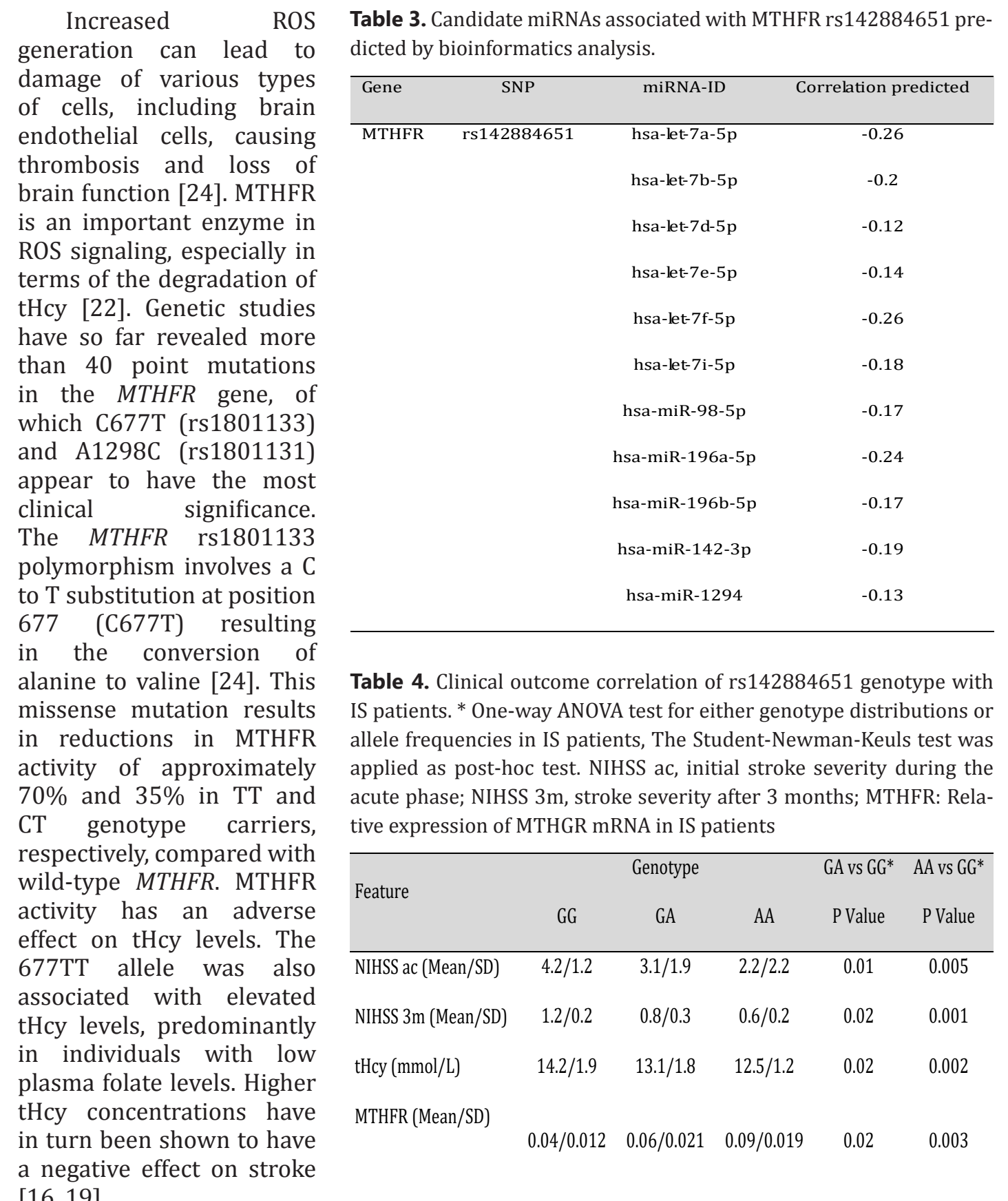


levels. The MTHFR rs142884651 SNP was also associated with decreased serum tHcy and IS outcome. The results of the current study thus demonstrated the importance of the MTHFR rs142884651 SNP in MTHFR expression, let-7f and miR-196a binding, the development of IS, and short-term outcomes in IS patients.

This study had some limitations. We did not further assess the effects of the miRNAs on the regulation of IS risk and outcome. In addition, our study was conducted in a West Chinese Han population, and may also have been affected by type 1 statistical errors. The results should therefore be confirmed in independent samples or samples from another ethnic population, and should also be verified in future studies with larger sample sizes.

\section{Conclusion}

In summary, we demonstrated that the MTHFR rs142884651 SNP is associated with a decreased risk of IS and may act as a biomarker of short-term outcome in IS patients.

\section{Disclosure Statement}

The authors declare that there are no conflicts of interest in this work.

\section{References}

1 Kehrel BE, Fender AC: Resolving Thromboinflammation in the Brain After Ischemic Stroke? Circulation 2016;133:2128-2131.

- Nordestgaard BG: Triglyceride-Rich Lipoproteins and Atherosclerotic Cardiovascular Disease: New Insights From Epidemiology, Genetics, and Biology. Circ Res 2016;118:547-563.

-3 Global Burden of Disease Study C: Global, regional, and national incidence, prevalence, and years lived with disability for 301 acute and chronic diseases and injuries in 188 countries, 1990-2013: a systematic analysis for the Global Burden of Disease Study 2013 Lancet 2015;386:743-800.

-4 Akoudad S, Portegies ML, Koudstaal PJ, Hofman A, van der Lugt A, Ikram MA, Vernooij MW: Cerebral Microbleeds Are Associated With an Increased Risk of Stroke: The Rotterdam Study. Circulation 2015;132:509-516.

5 Courties G, Herisson F, Sager HB, Heidt T, Ye Y, Wei Y, Sun Y, Severe N, Dutta P, Scharff J, Scadden DT, Weissleder R, Swirski FK, Moskowitz MA, Nahrendorf M: Ischemic stroke activates hematopoietic bone marrow stem cells. Circ Res 2015;116:407-417.

6 Rocha SF, Schiller M, Jing D, Li H, Butz S, Vestweber D, Biljes D, Drexler HC, Nieminen-Kelha M, Vajkoczy P, Adams S, Benedito R, Adams RH: Esm1 modulates endothelial tip cell behavior and vascular permeability by enhancing VEGF bioavailability. Circ Res 2014;115:581-590.

7 Donnan GA, Fisher M, Macleod M, Davis SM: Stroke. Lancet 2008;371:1612-1623.

8 Moll S, Varga EA: Homocysteine and MTHFR Mutations. Circulation 2015;132:e6-9.

-9 Elanchezhian R, Palsamy P, Madson CJ, Lynch DW, Shinohara T: Age-related cataracts: homocysteine coupled endoplasmic reticulum stress and suppression of Nrf2-dependent antioxidant protection. Chem Biol Interact 2012;200:1-10.

10 Zhang D, Fang P, Jiang X, Nelson J, Moore JK, Kruger WD, Berretta RM, Houser SR, Yang X, Wang H: Severe hyperhomocysteinemia promotes bone marrow-derived and resident inflammatory monocyte differentiation and atherosclerosis in LDLr/CBS-deficient mice. Circ Res 2012;111:37-49.

11 Wang X, Cui L, Joseph J, Jiang B, Pimental D, Handy DE, Liao R, Loscalzo J: Homocysteine induces cardiomyocyte dysfunction and apoptosis through p38 MAPK-mediated increase in oxidant stress. J Mol Cell Cardiol 2012;52:753-760.

12 Zhong C, Xu T, Xu T, Peng Y, Wang A, Wang J, Peng H, Li Q, Geng D, Zhang D, Zhang Y, Zhang Y, Gao X, He J, Groups CI: Plasma Homocysteine and Prognosis of Acute Ischemic Stroke: a Gender-Specific Analysis From CATIS Randomized Clinical Trial. Mol Neurobiol 2016;10.1007/s12035-016-9799-0 
13 Liu C, Zhang Y, Chen H, Jiang L, Xiao D: Function analysis of rs9589207 polymorphism in miR-92a in gastric cancer. Tumour Biol 2015;10.1007/s13277-015-4288-z

14 Qin X, Li Y, Yuan H, Xie D, Tang G, Wang B, Wang X, Xu X, Xu X, Hou F: Relationship of MTHFR gene 677C --> T polymorphism, homocysteine, and estimated glomerular filtration rate levels with the risk of new-onset diabetes. Medicine (Baltimore) 2015;94:e563.

15 Yildiz SH, Ozdemir Erdogan M, Solak M, Eser O, Arikan Terzi ES, Eser B, Kocabas V, Aslan A: Lack of association between the methylenetetrahydropholate reductase gene A1298C polymorphism and neural tube defects in a Turkish study group. Genet Mol Res 2016;15:

-16 Yue YH, Bai XD, Zhang HJ, Li YM, Hu L, Liu LY, Mao JP, Yang XY, Dila NM: Gene Polymorphisms Affect the Effectiveness of Atorvastatin in Treating Ischemic Stroke Patients. Cell Physiol Biochem 2016;39:630-638.

17 Tasdemir S, Erdem HB, Sahin I, Ozel L, Ozdemir G, Eroz R, Tatar A: Correlation with Platelet Parameters and Genetic Markers of Thrombophilia Panel (Factor II g.20210G>A, Factor V Leiden, MTHFR (C677T, A1298C), PAI-1, beta-Fibrinogen, Factor XIIIA (V34L), Glycoprotein IIIa (L33P)) in Ischemic Strokes. Neuromolecular Med 2016;18:170-176.

18 Ho GY, Eikelboom JW, Hankey GJ, Wong CR, Tan SL, Chan JB, Chen CP: Methylenetetrahydrofolate reductase polymorphisms and homocysteine-lowering effect of vitamin therapy in Singaporean stroke patients. Stroke 2006;37:456-460.

19 He W, Lu M, Li G, Sun Z, Liu D, Gu L: Methylene Tetrahydrofolate Reductase (MTHFR) rs868014 Polymorphism Regulated by miR-1203 Associates with Risk and Short Term Outcome of Ischemic Stroke. Cell Physiol Biochem 2017;41:701-710.

20 Wang Y, Zhou L, Chen J, Li J, He L, Wu P, Wang M, Tong N, Zhang Z, Fang Y: Association of the 3'UTR FOXO3a polymorphism rs4946936 with an increased risk of childhood acute lymphoblastic leukemia in a Chinese population. Cell Physiol Biochem 2014;34:325-332.

21 Tang J, Zhuo H, Zhang X, Jiang R, Ji J, Deng L, Qian X, Zhang F, Sun B: A novel biomarker Linc00974 interacting with KRT19 promotes proliferation and metastasis in hepatocellular carcinoma. Cell Death Dis 2014;5:e1549.

22 Chen Q Qin R, Fang Y, Li H, Liu Y: A Functional Variant at the miR-214 Binding Site in the Methylenetetrahydrofolatereductase Gene Alters Susceptibility to Gastric Cancer in a Chinese Han Population. Cell Physiol Biochem 2015;36:622-630.

23 Chan PH: Reactive oxygen radicals in signaling and damage in the ischemic brain. J Cereb Blood Flow Metab 2001;21:2-14.

24 Kim JO, Park HS, Ryu CS, Shin JW, Kim J, Oh SH, Kim OJ, Kim NK: Interplay between 3'-UTR polymorphisms in the methylenetetrahydrofolate reductase (MTHFR) gene and the risk of ischemic stroke. Sci Rep 2017;7:12464.

25 Min SK, Jung SY, Kang HK, Park SA, Lee JH, Kim MJ, Min BM: Functional diversity of miR-146a-5p and TRAF6 in normal and oral cancer cells. Int J Oncol 2017;51:1541-1552.

-26 Bartel DP: MicroRNAs: genomics, biogenesis, mechanism, and function. Cell 2004;116:281-297. 